\title{
Cyclophosphamide-induced HCN1 channel upregulation in interstitial Cajal-like cells leads to bladder hyperactivity in mice
}

\author{
Qian Liu, Zhou Long, Xingyou Dong, Teng Zhang, Jiang Zhao, Bishao Sun, Jingzhen Zhu, Jia Li,
} Qingqing Wang, Zhenxing Yang, Xiaoyan $\mathrm{Hu}$ and Longkun Li

Hyperpolarization-activated cyclic nucleotide-gated (HCN) channels are confirmed to be expressed in bladder interstitial Cajal-like cells (ICC-LCs), but little is known about their possible role in cystitis-associated bladder dysfunction. The present study aimed to determine the functional role of $\mathrm{HCN}$ channels in regulating bladder function under inflammatory conditions. Sixty female wild-type C57BL/6J mice and sixty female HCN1-knockout mice were randomly assigned to experimental and control groups, respectively. Cyclophosphamide (CYP)-induced cystitis models were successfully established in these mice. CYP treatment significantly enhanced $\mathrm{HCN}$ channel protein expression and $I_{\mathrm{h}}$ density and significantly altered bladder HCN1 channel regulatory proteins. Carbachol (CCH) and forskolin (FSK) exerted significant effects on bladder ICC-LC $\left[\mathrm{Ca}^{2+}\right]_{\mathrm{i}}$ in CYP-treated wild-type (WT) mice, and HCN1 channel ablation significantly decreased the effects of CCH and FSK on bladder ICC-LC $\left[\mathrm{Ca}^{2+}\right]_{\mathrm{i}}$ in both naive and CYP-treated mice. CYP treatment significantly potentiated the spontaneous contractions and $\mathrm{CCH}(0.001-10 \mu \mathrm{m})$-induced phasic contractions of detrusor strips, and HCN1 channel deletion significantly abated such effects. Finally, we demonstrated that the development of CYP-induced bladder overactivity was reversed in HCN $1-I-$ mice. Taken together, our results suggest that CYP-induced enhancements of HCN1 channel expression and function in bladder ICC-LCs are essential for cystitis-associated bladder hyperactivity development, indicating that the HCN1 channel may be a novel therapeutic target for managing bladder hyperactivity.

Experimental \& Molecular Medicine (2017) 49, e319; doi:10.1038/emm.2017.31; published online 21 April 2017

\section{INTRODUCTION}

Inflammation of the urinary bladder, cystitis, can disturb the storage function of the bladder and lead to a series of bothersome lower urinary tract symptoms (LUTS), such as urgency, frequency and urge incontinence, which are the components of overactive bladder $(\mathrm{OAB})$ syndrome. ${ }^{1,2}$ Interstitial cystitis/painful bladder syndrome (IC/PBS) is a special type of cystitis that is estimated to affect $3-6 \%$ of women in the United States $^{3}$ and is characterized by urinary frequency, urgency, pelvic pain and other types of discomfort. ${ }^{4}$ In the clinic, frequency or urgency is a far more common presentation than bladder pain in patients with IC/PBS. ${ }^{5}$ The animal model of chemically induced cystitis is most commonly used to mimic the symptoms of IC/PBS. ${ }^{6}$ Cyclophosphamide (CYP)-induced cystitis is an acknowledged experimental model of bladder inflammation and dysfunction. ${ }^{7}$ A previous study demonstrated that mice with chronic CYP-induced cystitis exhibited a suite of OAB syndrome symptoms. ${ }^{8}$ Furthermore,
CYP is a common anticancer agent and can induce urinary bladder inflammation, leading to irritative LUTS in cancer patients. ${ }^{9}$ Hence, using the mouse model of CYP-induced cystitis, we attempted to elucidate the mechanisms underlying the development of bladder hyperactivity in CYP-induced cystitis-associated bladder dysfunction.

Interstitial Cajal cells (ICCs) were first identified in the gut and have been confirmed to be gastrointestinal pacemakers involved in conducting slow-wave activity and driving peristalsis. ${ }^{10}$ In recent years, several studies have discovered that interstitial Cajal-like cells (ICC-LCs) are present in the bladders of humans and animals. ${ }^{11}$ Various ion channels are expressed in the membranes of bladder ICC-LCs and influence bladder ICC-LC electrophysiologic activity. ${ }^{12-14}$ McCloskey and Gurney demonstrated that guinea pig bladder ICC-LCs are spontaneously active and can exhibit $\mathrm{Ca}^{2+}$ wave firing upon cholinergic stimulation. These authors suggested that bladder ICC-LCs can act as pacemakers to regulate detrusor 
contractility or as intermediaries in the transmission of nerve signals to detrusor smooth muscle cells. ${ }^{15}$ Evidence indicates that changes in bladder ICC-LC quantity and function are associated with pathological conditions, such as obstructed bladder, diabetic bladder and neurogenic bladder. ${ }^{16}$ However, only bladder ICC-LC distributions and phenotypes are altered in IC/PBS. ${ }^{17}$ Further research is needed to address the functional alterations of bladder ICC-LCs that occur during cystitis to exploit the opportunity to develop new therapeutic strategies to treat this condition.

Hyperpolarization-activated current, $I_{\mathrm{h}}$, was first identified in sinoatrial node tissue in 1976 and was thought to be the pacemaker current. Hyperpolarization-activated cyclic nucleotide $(\mathrm{HCN})$ channels, four subtypes (HCN1-4) of which exist, were found to underlie $I_{\mathrm{h}}$ in the brain and heart in the 1990s. ${ }^{18}$ HCN channels have proven to play a crucial role in many physiological processes, such as dendritic integration, synaptic transmission, neuronal oscillation and cardiac rhythmicity. ${ }^{19} \mathrm{HCN}$ channels are also involved in regulating pacemaker activity in the colonic interstitial cells of Cajal. ${ }^{20}$ Furthermore, HCN channel expression and function are influenced by numerous small molecules, protein kinases and interacting proteins. In particular, in the nervous system, several proteins (including Filamin A and NEDD4L) have been shown to act as auxiliary proteins involved in regulating HCN1 channel voltage gating, kinetics and cell surface expression. ${ }^{18}$

Our previous studies showed that all four HCN subtypes are expressed exclusively in ICC-LCs and that the HCN1 channel is the predominant channel expressed in the bladder. HCN channels were thought to be the key modulators of bladder ICC-LCs with respect to the conduction of bladder pacemaker activity. ${ }^{12}$ In addition, HCN channel expression and function were significantly altered in the bladder ICC-LCs of diabetic cystopathy and detrusor overactive (DO) bladders with partial bladder outlet obstruction (PBOO), ${ }^{21,22}$ suggesting that HCN channels in bladder ICC-LCs are involved in regulating bladder function in physiological and pathological conditions. To our knowledge, the role of HCN channels in bladder ICC-LCs in cystitis remains unknown. The current study demonstrated that CYP can enhance HCN channel expression and function in bladder ICC-LCs by disturbing HCN channel regulatory proteins and also clarified the functional role of $\mathrm{HCN} 1$ channels in regulating bladder function in both physiological and inflammatory conditions using HCN1-knockout mice.

\section{MATERIALS AND METHODS}

\section{Clinical specimens}

Five fresh bladder tissue specimens obtained via radical cystectomy from patients undergoing chemotherapy due to bladder cancer served as the experimental group, and five fresh normal bladder tissue specimens obtained via radical cystectomy from patients who were not undergoing chemotherapy served as the control group. All the samples were collected in the Department of Urology, Second Affiliated Hospital, Third Military Medical University, China, and all the specimens were snap-frozen in liquid nitrogen. All experiments were conducted in accordance with the approved guidelines and were approved by the Institutional Review Board of the Third Military Medical University, China.

\section{Animals}

Experiments were conducted on female wild-type C57BL/6J mice and homozygous HCN1-knockout mice (HCN1 - / -, B6.129S$\mathrm{Hcn}^{\mathrm{tm} 2 \mathrm{Kndl} / J}$, Jackson Laboratory, Stock \#016566). All the study mice were aged 10-12 weeks and weighed $18-25 \mathrm{~g}$. The mice were housed in cages under a standard 12-h/12-h light-dark cycle, with free access to food and water. Sixty wild-type C57BL/6J mice and $60 \mathrm{HCN} 1-/-$ mice were randomly assigned to control and experimental groups, respectively. All animal experiments were performed in accordance with the Guide for Care and Use of Laboratory Animals promulgated by the National Institutes of Health and were approved by the Research Council and Animal Care and Use Committee of the Third Military Medical University, China.

\section{Induction of CYP-induced cystitis}

Cystitis was induced via intraperitoneal injections of cyclophosphamide (CYP; Sigma, St Louis, MO, USA), as described by others. ${ }^{8}$ CYP $\left(80 \mathrm{mg} \mathrm{kg}^{-1}\right)$ or vehicle (sterile saline) was administered every other day for a period of 7 days. The mice were killed $24 \mathrm{~h}$ after the fourth CYP or vehicle injection.

\section{Hematoxylin and eosin staining}

The dissected bladders were fixed with $4 \%$ paraformaldehyde (Boster, Wuhan, China) overnight before being dehydrated and embedded in paraffin, and $4-\mu \mathrm{m}$ sections were prepared for hematoxylin and eosin (H\&E) staining, which was performed following a standard protocol. ${ }^{23}$ Briefly, the sections were sequentially stained in hematoxylin and $1 \%$ eosin solution for 30 and $10-30$ s, respectively, with agitation. The sections were then dehydrated in ethanol and immobilized with mounting medium before being viewed and photographed under an optical microscope (Olympus, Tokyo, Japan). Urothelial thickness was measured using Image-pro plus 6.0 software (Media Cybernetics, Silver Spring, MD, USA).

\section{Western blotting}

For western blotting, total protein was extracted from mouse bladders and clinical specimens using RIPA lysis buffer (Beyotime, Shanghai, China), and protein concentrations were measured using a Bio-Rad DC Protein Assay Kit (Bio-Rad, Hercules, CA, USA). Fifty micrograms of protein was then loaded onto SDS-PAGE gels and separated before being transferred to PVDF membranes (Merck Millipore, Darmstadt, Germany). After being blocked with $5 \%$ bovine serum albumin dissolved in Tris-buffered saline for $2 \mathrm{~h}$, the membranes were incubated with primary antibodies to the following proteins overnight at $4{ }^{\circ} \mathrm{C}$ : IL-6 (GeneTex, San Antonio, TX, USA, GTX110527, 1:1000), TNF- $\alpha$ (GeneTex, GTX110520, 1:1000), HCN1 (Abcam, Cambridge, UK, ab84816, 1:1000), HCN2 (Abcam, ab65704, 1:1000), HCN3 (Abcam, ab84818, 1:1000), HCN4 (Abcam, ab69054, 1:1000), Filamin A (Cell Signaling Technology, Beverly, MA, USA, 4762, 1:1000), NEDD4L (Cell Signaling Technology, 4013, 1:1000), $\alpha$-tubulin (Beyotime, AT819, 1:1000) and GAPDH (Beyotime, AG019, 1:1000). Horseradish peroxidase-conjugated species-specific secondary antibodies (Zhongshan Co., Beijing, China, ZB-2301, ZB-2305, 1:5000) were used to detect the above primary antibodies. The proteins were visualized using ECL Substrate 
Table 1 Primers designed for quantitative RT-PCR

\begin{tabular}{|c|c|c|c|}
\hline Gene & Species & Primer sequence & Accession number \\
\hline C-kit & Mouse & $\begin{array}{l}\text { F: 5'-TTCCGCCTTTACGAGCACACA-3' } \\
\text { R: 5'-CCCGCCCTTCCCTTCTCCTC-3' }\end{array}$ & Y00864 \\
\hline Myosin & Mouse & $\begin{array}{l}\text { F: 5'-ACCCGGCAGAAGCTCAATGTG-3' } \\
\text { R: 5'-GCCCTCCATCTCTTTCTGTAACC-3' }\end{array}$ & NM_013607 \\
\hline GAPDH & Mouse & $\begin{array}{l}\text { F: 5'-GGCCGGTGCTGAGTATGTCGTG-3' } \\
\text { R: 5'-GGGGGGCTAAGCAGTTGGTGG-3' }\end{array}$ & NM_001289726 \\
\hline
\end{tabular}

Abbreviations: F, forward; GAPDH, glyceraldehyde phosphate dehydrogenase; PGP9.5, protein gene product 9.5; R, reverse.

(Millipore, Billerica, MA, USA) and detected using an Image Quant LAS-4000 BioImaging System (GE Healthcare, Stockholm, Sweden).

\section{Immunofluorescence and bladder ICC-LC cell counting}

The dissected bladders were cut into 6 - $\mu \mathrm{m}$ sections on a freezing microtome after being snap-frozen. These tissue sections were then fixed in $4 \%$ paraformaldehyde (Boster) for $30 \mathrm{~min}$ and washed in PBS (10 min $\times 3$ times). After being preincubated with immunostaining blocking buffer (Beyotime) to block nonspecific epitopes for $60 \mathrm{~min}$, the tissue sections were incubated overnight at $4{ }^{\circ} \mathrm{C}$ with c-kit primary antibodies (Santa Cruz, Dallas, TX, USA, sc-1494, 1:50), followed by incubation with the appropriate fluorescence-conjugated Alexa 488 mouse anti-goat IgG (Bioss, Beijing, China, bs-0294M, 1:200). The tissue sections were subsequently incubated with 2-(4amidinophenyl)-6-indolecarbamidine dihydrochloride (DAPI, Beyotime) to mark cell nuclei. All incubation steps were followed by three 10-min PBS washes. Negative control sections were prepared by omitting the primary antibody. All tissue sections were viewed using a laser-scanning confocal microscope (Leica, Wetzlar and Mannheim, Germany). For quantitative assessment of the bladder ICC-LCs, the numbers of c-kit-positive cells in each group were counted. We ultimately excluded c-kit-positive cells with round shapes from the final counting results to eliminate interference from mast cells, as reported previously. ${ }^{24}$ Five bladders were obtained from each group and three sections were prepared for each bladder tissue. Each section was scanned in three random high-power fields (HPFs, $\times 800$ magnification) and bladder ICC-LCs in the detrusor were counted. The averaged data obtained by three researchers who were blinded to the group information represented the quantities of bladder ICC-LCs in each HPF.

\section{Preparation of isolated bladder ICC-LCs}

The entire bladder was removed aseptically from each killed mouse and washed thrice in sterile PBS solution. The bladder was then transferred into sterile $\mathrm{Ca}^{2+}$-free Hank's solution (Boster), and the urothelium was dissected away. After being minced, the bladder fragments were incubated for $5 \mathrm{~min}$ at $37^{\circ} \mathrm{C}$ in $2 \mathrm{ml}$ of enzyme solution containing the following reagents $\left(\mathrm{mg} \mathrm{ml}^{-1}\right)$ : 1.0 type II collagenase, 1.0 BSA, and 1.0 trypsin inhibitor (all were purchased from Sigma). An equal volume of RMPI-1640 medium (HyClone, Logan, UT, USA) containing 10\% fetal bovine serum (Gibco-Life Technologies, Grand Island, NY, USA) was applied to terminate digestion. After being resuspended, the cells were collected using a 200-mesh cell strainer. To ensure maximum bladder fragment utilization and collect more cells, we repeated the digestive process several times. The cells were ultimately plated onto sterile polylysin-coated glass coverslips and cultured at $37^{\circ} \mathrm{C}$ in a $95 \%$ $\mathrm{O}_{2}$ and $5 \% \quad \mathrm{CO}_{2}$ incubator in RMPI-1640 medium containing $10 \%$ fetal bovine serum and supplemented with $1 \%$ antibiotics/ antimycotics (Beyotime).

\section{Patch-clamp experiments}

The patch-clamp technique was tested on bladder ICC-LCs that had been cultured for 2-3 days. These cells were identified based on their distinctive morphology, as they were stellate- or spindle-shaped, with multiple branches. ${ }^{25}$ Electrodes with approximate resistances of 4-6 M $\Omega$ were pulled from a Model P-97 Flaming/Brown Micropipette Puller (Sutter Instrument Co., Novato, CA, USA). $I_{\mathrm{h}}$ was measured in the whole-cell voltage-clamp configuration. An intracellular pipette solution containing the following compounds was used for the recordings (in $\mathrm{mm}$ ): $130 \mathrm{~K}$ aspartate, $0.1 \mathrm{Na}_{2} \mathrm{GTP}, 5 \mathrm{Na}_{2} \mathrm{ATP}$, $2 \mathrm{MgCl}_{2}, 5 \mathrm{CaCl}_{2}, 11$ EGTA, 10 HEPES, pH 7.2 with KOH. The bath solution contained the following compounds (in mM): $140 \mathrm{NaCl}$, $1.2 \mathrm{MgCl}_{2}, 5.4 \mathrm{KCl}, 1.8 \mathrm{CaCl}_{2}, 10$ glucose, 5 HEPES, pH 7.4 with $\mathrm{NaOH}$. To pharmacologically isolate $I_{\mathrm{h}}, 1 \mathrm{~mm} \mathrm{BaCl}_{2}$ and $0.001 \mathrm{~mm}$ TTX (Tocris Bioscience, Bristol, UK) were added to the bath solution. ZD7288 (50 $\mu \mathrm{M}$, Sigma) was administered to confirm the specificity of $I_{\mathrm{h}}$ further. $I_{\mathrm{h}}$ was amplified and recorded on a HEKA EPC10 USB amplifier (HEKA Elektronik, Heidelberg, Germany) and filtered at a threshold frequency of $2.9 \mathrm{kHz}$. The cells were clamped at a holding potential of $-60 \mathrm{mV}$, followed by a step potential ranging from -60 to $-120 \mathrm{mV}$ in decrements of $10 \mathrm{mV}$. The cells then underwent a voltage jump to $-120 \mathrm{mV}$, at which the $\mathrm{HCN}$ channels were fully activated. Data were analyzed using FitMaster software (HEKA Elektronik), and $I_{\mathrm{h}}$ was normalized to cell capacitance.

\section{Single-cell RT-PCR}

To confirm the reliability of the isolated bladder ICC-LCs further, we performed single-cell RT-PCR, as previously described. ${ }^{26}$ After electrophysiological testing, single-bladder ICC-LCs were sucked into a recording pipette individually by applying negative pressure. Each cell was then immediately expelled from the pipette into a $1.5-\mathrm{ml}$ tube containing NP-40 lysis buffer and an RNAase inhibitor (both were purchased from Beyotime) and centrifuged for $30 \mathrm{~s}$ at 1000 r.p.m. and 
$4{ }^{\circ} \mathrm{C}$. Each cell was subsequently lysed on ice for $20 \mathrm{~min}$. cDNAs were synthesized using a Sensiscript RT Kit (Qiagen, Hilden, Germany), according to the manufacturer's instructions. The primers used in our study are listed in Table 1 . The thermocycling program consisted of the following steps: $94^{\circ} \mathrm{C}$ for $1 \mathrm{~min}, 60^{\circ} \mathrm{C}$ for $30 \mathrm{~s}$ and $72^{\circ} \mathrm{C}$ for $1 \mathrm{~min}$ (40 cycles). Negative control reactions were conducted using the same amount of RNA, without reverse transcription. The PCR products were visualized via $2 \%$ agarose gel electrophoresis followed by GoldView (SBS Genetech Co., Beijing, China) staining.

\section{Measurement of intracellular calcium ion concentrations $\left(\left[\mathrm{Ca}^{2+}\right]_{\mathrm{i}}\right)$}

After routine culturing for 2-3 days, the primary isolated bladder ICC-LCs were washed with Hank's solution (Boster) for $5 \mathrm{~min}$ and then incubated with Fluo-4 AM $(10 \mu \mathrm{M}$, Molecular Probes, Eugene, OR, USA) for $30 \mathrm{~min}$ at $37^{\circ} \mathrm{C}$. After incubation, the bladder ICC-LCs were washed with Hank's solution ( $5 \mathrm{~min} \times 3$ times), and $\mathrm{Ca}^{2+}$ imaging was conducted under a laser-scanning confocal microscope (Leica) at an emission wavelength of $488 \mathrm{~nm}$. Bladder ICC-LCs were identified based on their distinctive morphology, as described above. The effects of forskolin (FSK, $50 \mu \mathrm{m}$, Sigma) and carbachol $(\mathrm{CCH}$, $1 \mu \mathrm{M}$, Merck Millipore, Darmstadt, Germany) on bladder ICC-LC real-time $\left[\mathrm{Ca}^{2+}\right]_{\mathrm{i}}$ were analyzed. ZD7288 $(50 \mu \mathrm{M}$, Sigma $)$ was administered to confirm the authenticity of the isolated bladder ICC-LCs further. The final data were presented as relative fluorescence intensities $(\mathrm{RFI}=F 1 / F 0$, where $F 1$ is the real-time fluorescence intensity after drug administration, and $F 0$ is the baseline fluorescence intensity before drug administration).

\section{Contractility studies}

After gently denuding the urothelial layer, we longitudinally cut the mouse bladders into strips $\sim 1 \times 2 \times 4 \mathrm{~mm}$ in size. Each strip was mounted in a 15-ml tissue bath filled with aerated Kreb's solution ( $95 \% \mathrm{O}_{2}$ and $5 \% \mathrm{CO}_{2}, 37^{\circ} \mathrm{C}$ ), containing the following compounds (in mM): $119 \mathrm{NaCl}, 4.7 \mathrm{KCl}, 1.2 \mathrm{KH}_{2} \mathrm{PO}_{4}, 1.2 \mathrm{MgSO}_{4} \cdot 7 \mathrm{H}_{2} \mathrm{O}$, $25 \mathrm{NaHCO}_{3}, 2.5 \mathrm{CaCl}_{2}$ and 11 glucose, adjusted to $\mathrm{pH} 7.4$ with $\mathrm{NaOH}$. Each strip was suspended vertically between two curved hooks. One hook was fixed on the bottom of the bath, and the other hook was connected to a stretch transducer (Chengyi Co., Chengdu, China), which was calibrated before every experiment. After equilibrating for $30 \mathrm{~min}$, each strip was stretched to $0.2 \mathrm{~g}$ of tension and allowed to stabilize for $10 \mathrm{~min}$ before beginning the experimental protocols. Stretch transducer output was amplified and recorded using an RM6240C (Chengyi Co.). Gradient doses of CCH (0.001-10 $\mu \mathrm{M}$, purchased from Merck Millipore) were added to the bathing solution at 6-min intervals. After a round of $\mathrm{CCH}$ dosing, the drugs were washed out by fresh Kreb's solution with a temperature of $37^{\circ} \mathrm{C}$.

\section{Urodynamic measurements in unconscious mice}

Urodynamic measurements in unconscious mice were performed as previously described, ${ }^{27}$ with some modifications. The experiments were conducted under urethane anesthesia $\left(1 \mathrm{~g} \mathrm{~kg}^{-1}\right.$ body weight, intraperitoneally). A PE-10 polyethylene catheter (Smiths Medical, Saint Paul, MN, USA) was introduced into the bladder through the external urethral orifice and connected to a three-way valve linked to a pressure transducer (Chengyi Co.) and an infusion pump (AVI 270, Minnesota Mining \& Manufacturing Company, Saint Paul, MN, USA). The pressure transducer was calibrated before each experiment. Each bladder was infused with room temperature saline at a constant rate $\left(1 \mathrm{ml} \mathrm{h}^{-1}\right)$ and continuous urodynamic curves were visualized using a multi-channel signal processing system (RM6240C, Chengyi Co.). The following urodynamic parameters were analyzed: intercontractile interval (ICI), basal pressure (BP), maximum bladder pressure (MBP) and nonvoiding contractions (NVCs). NVCs are defined as increases that possess a minimal amplitude of $1 \mathrm{~cm} \mathrm{H}_{2} \mathrm{O}$ and a minimal duration of $3 \mathrm{~s}$ in intravesical pressure during cystometry, not associated with micturition. ${ }^{27,28}$

\section{Statistical analysis}

All experimental data are presented as the mean \pm s.d. Statistical analyses were performed with SPSS 13.0 software (SPSS Inc., Chicago, IL, USA), and the significance of the differences between individual groups was calculated using unpaired two-tailed Student's $t$-tests or one-way ANOVA. Statistical significance was defined as a conventional $P$-value $<0.05$. All experiments were performed with a minimum of three independent replications.

\section{RESULTS}

\section{Development of cyclophosphamide-induced cystitis in} WT and HCN1 - / - mice

Upon systemic application, the chemotherapeutic agent cyclophosphamide is partially metabolized to acrolein by the liver. Acrolein subsequently accumulates in the bladder, resulting in hemorrhagic cystitis. ${ }^{1}$ Multiple injections of low doses of CYP can lead to chronic CYP-induced cystitis and chronic CYP-induced detrusor overactivity in mice. ${ }^{8}$ To investigate the role of the HCN1 channel in CYP-induced cystitis, we first evaluated the inflammation levels of bladders in CYP-treated WT and HCN1 - / - mice via H\&E staining. The bladder sections from both CYP-treated WT and HCN1 - / - mice exhibited considerable edema in the lamina propria, with increased paracellular space (Figure 1a), and increased urothelial thickness (Figure 1b). Furthermore, the inflamed bladders from CYP-treated WT and HCN1 - / mice were heavier than the bladders from vehicle-treated (naive) mice (Figure 1c). In addition, we detected the levels of two inflammation-associated cytokines (IL- 6 and TNF- $\alpha$ ) by western blotting to determine whether inflammation was present. The expression levels of both IL- 6 and TNF- $\alpha$ in the bladders of CYP-treated mice were almost twice as high as those in the bladders of naive mice in both genotypes (Figure 1d and f). Based on these results, we concluded that CYP-treated WT and HCN1 - / - mice exhibited similar inflammatory responses. Moreover, significant elevations in the expression levels of IL- 6 and TNF- $\alpha$ were noted in the bladder samples of patients undergoing chemotherapy, revealing the presence of chemotherapeutic agent-induced inflammation, which was suggested to be similar to CYP-induced cystitis (Figure 1e and g).

\section{Enhanced $\mathrm{HCN}$ channel protein expression in CYP-induced cystitis}

Using western blotting, we detected alterations in the protein expression levels of the four HCN subtypes in the bladders of CYP-treated mice. CYP treatment resulted in increased protein expression levels of all four HCN subtypes. Meanwhile, no significant differences in the protein expression levels of 
a

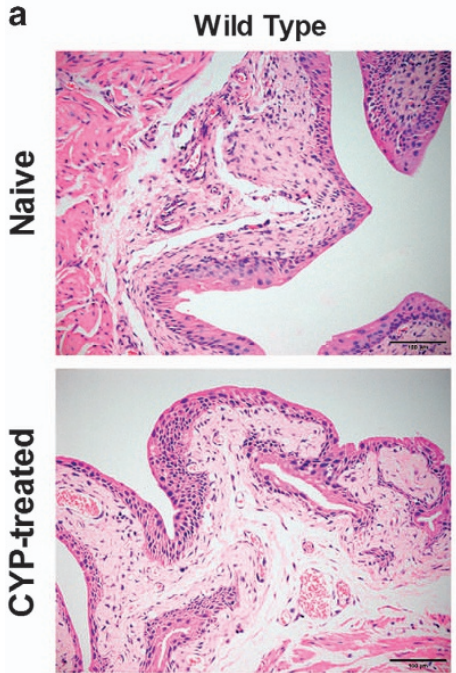

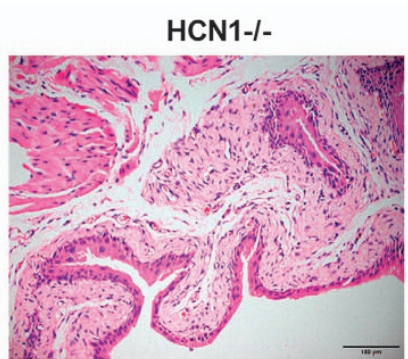

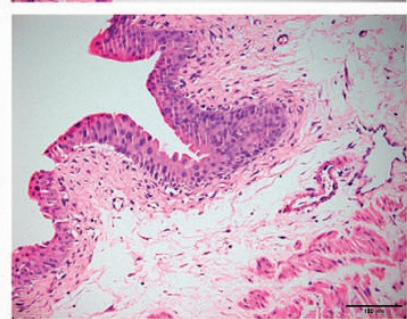

d

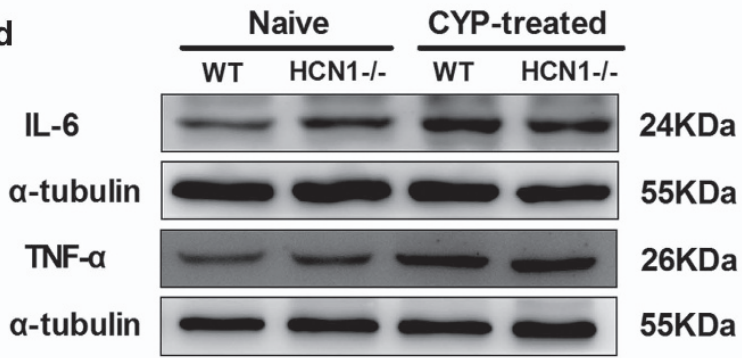

55KDa f

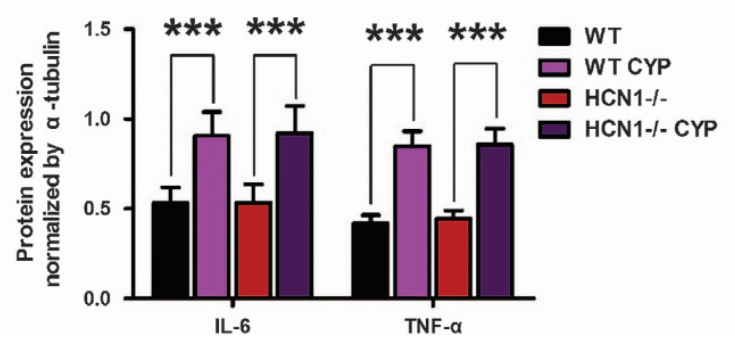

b
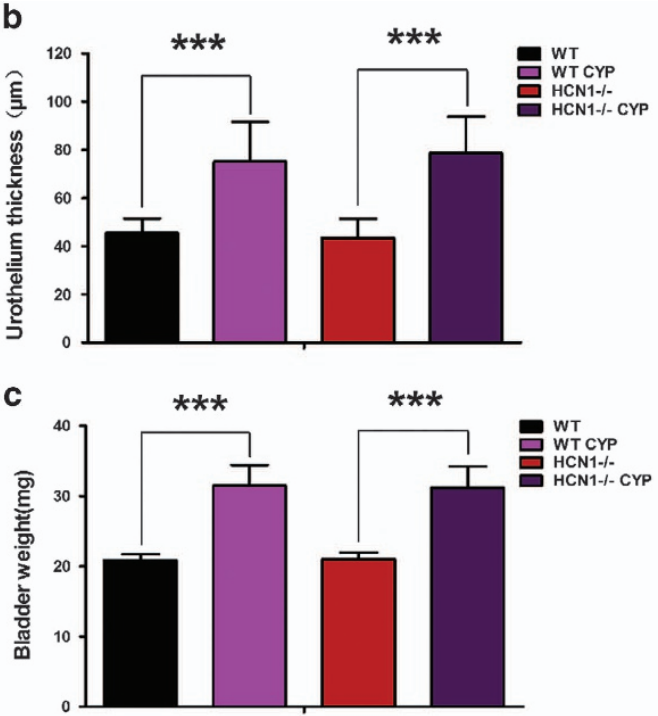

e

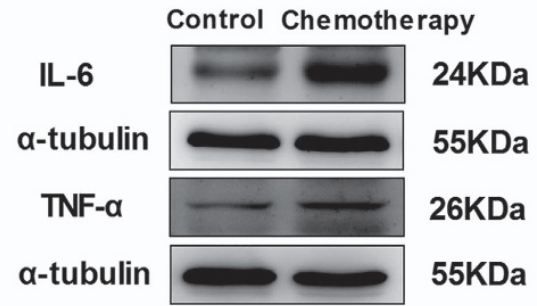

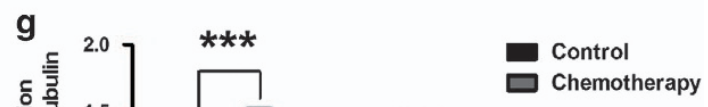

Figure 1 Cyclophosphamide (CYP)-induced cystitis in WT and HCN1-/- mice. (a) Characteristic inflammation was detected in histological bladder sections of CYP-treated WT and HCN1-/- mice. Urothelial thickness (b) and bladder weight (c) were significantly increased in both CYP-treated WT and HCN1-/- mice (data represent the mean \pm s.d., $N=10,{ }^{* * *} P<0.001$, versus naive mice). (d, f) IL-6 and TNF- $\alpha$ protein expression levels in the bladders of both WT and HCN1-/ - mice were significantly elevated when these mice were treated with CYP (data represent the mean \pm s.d., $N=6,{ }^{* *} P<0.001$, versus naive mice). (e, g) IL-6 and TNF- $\alpha$ protein expression levels were significantly enhanced in the bladder samples of patients undergoing chemotherapy (data represent the mean $\pm \mathrm{S}$.d., $N=5, * * * P<0.001$, versus control sample).

the four HCN subtypes were detected between WT and $\mathrm{HCN} 1-/-$ mice (Figure 2a and c). As shown in Figure 2b and $\mathrm{d}$, our clinical specimen results were consistent with our mouse model results. The protein expression levels of the four HCN subtypes in the bladder samples of patients undergoing chemotherapy were elevated compared with the protein expression levels of the four HCN subtypes in the bladder samples of control subjects.
Equal quantities of bladder ICC-LCs

We used immunofluorescence to count the average numbers of c-kit-positive ICC-LCs in single HPFs of bladder sections. There was no significant difference in the average number of c-kit-positive ICC-LCs between CYP-treated and naive mice of either genotype. Moreover, HCN1 channel knockout did not influence the average number of bladder ICC-LCs in either CYP-treated or naive mice (Figure $2 \mathrm{e}$ and $\mathrm{f}$ ). 


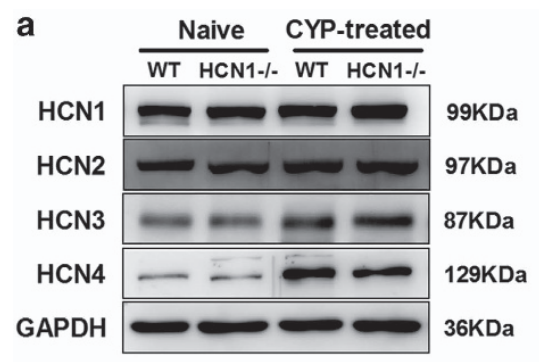

d
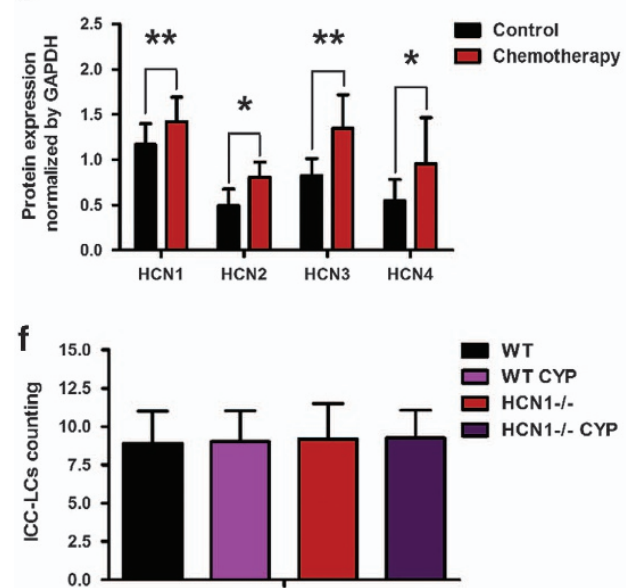

b

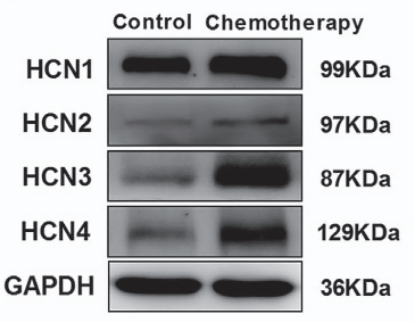

C

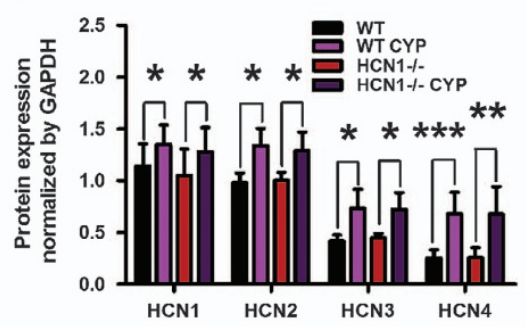

e

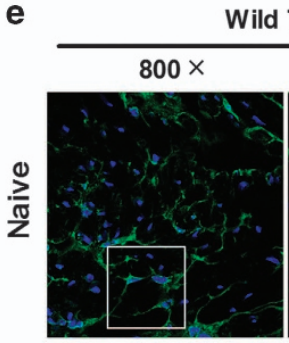

Wild Type
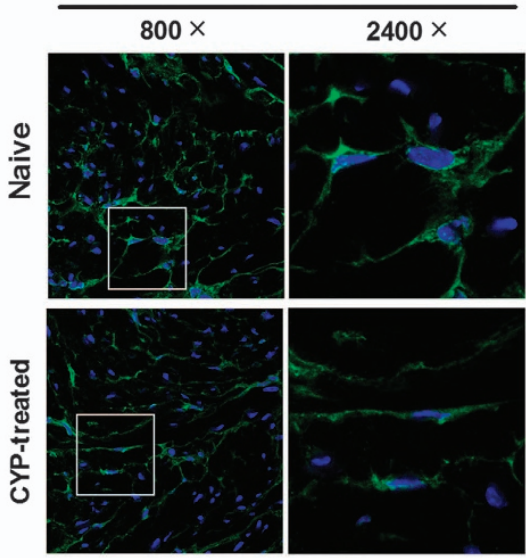

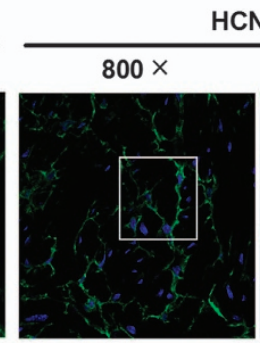

HCN1-I-

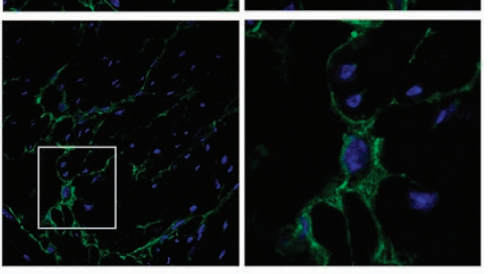

Figure 2 Increased HCN channel protein expression levels and equal quantities of bladder ICC-LCs in CYP-induced cystitis. (a, c) The protein expression levels of the four $\mathrm{HCN}$ subtypes were increased in the CYP-treated group (data represent the mean $\pm \mathrm{s} . \mathrm{d}$., $N=6$, ${ }^{*} P<0.05,{ }^{*} P<0.01$ and $* * * P<0.001$, versus naive mice). (b, d) The protein expression levels of the four HCN subtypes were significantly enhanced in the bladder samples of patients undergoing chemotherapy (data represent the mean $\pm \mathrm{s}$.d., $N=5,{ }^{*} P<0.05$ and ${ }^{*} P<0.01$, versus control sample). (e) Immunofluorescence labeling of bladder ICC-LCs in naive or CYP-treated WT and HCN1-/- mice. C-kit (green)-positive ICC-LCS were visualized under $800 \times$ magnification and ICC-LCS in white frames were showed under $\times 2400$ magnification. (f) There was no significant difference in the number of bladder ICC-LCs between each group (data represent the mean \pm s.d., $N=5$, all $P$-values $>0.05$ ).

Changes in the properties of $I_{\mathrm{h}}$ in bladder ICC-LCs

To test whether the properties of $I_{\mathrm{h}}$ were changed due to CYP treatment and HCN1 channel ablation, we conducted patch-clamp experiments. We applied the whole-cell patch clamp method to $I_{\mathrm{h}}$ recordings in isolated bladder ICC-LCs. Figure $3 \mathrm{a}$ shows the $I_{\mathrm{h}}$ of CYP-treated and naive mice in both genotypes. Application of a specific inhibitor of HCN channels, ZD7288 $(50 \mu \mathrm{M})$, reduced $I_{\mathrm{h}}$ amplitudes in all four groups, thereby confirming the specificity of these recorded $I_{\mathrm{h}}$ currents. As shown in Figure 3c, at the potential of $-120 \mathrm{mV}$, the $I_{\mathrm{h}}$ amplitude was highest in CYP-treated WT mice, as was the $\mathrm{HCN}$ channel gating rate. The $I_{\mathrm{h}}$ amplitude and $\mathrm{HCN}$ channel gating rate were significantly decreased in $\mathrm{HCN} 1-/-$ mice compared with WT mice. When normalized to cell capacitance, CYP treatment significantly increased $I_{\mathrm{h}}$ density in CYP-treated WT and HCN1 - / - mice, while HCN1 channel deletion led to significant reductions in $I_{\mathrm{h}}$ density in both CYP-treated and naive mice over the voltage range of -60 to $-120 \mathrm{mV}$ (Figure 3d-g). Our results indicate that at the potential of $-120 \mathrm{mV}, I_{\mathrm{h}}$ density was elevated by $\sim 60 \%$ in CYP-treated mice in both genotypes (Figure $3 \mathrm{~d}$ and e), and $I_{\mathrm{h}}$ density was decreased by $\sim 32 \%$ due to HCN1 channel knockout in both CYP-treated and naive mice (Figure $3 \mathrm{f}$ and g). We performed RT-PCR following electrophysiological testing, the results of which showed that only c-kit and GAPDH expression was visualized in the lanes (Figure $3 b$ ), thereby confirming the reliability of the isolated bladder ICC-LCs.

Altered Filamin A and NEDD4L protein expression levels in cystitis

We also validated that the protein expression levels of Filamin A and NEDD4L were significantly upregulated and downregulated, respectively, in CYP-induced cystitis (Figure 4a and c). Consistent with these findings, the protein expression level of Filamin A was noticeably increased, and the protein expression level of NEDD4L was significantly decreased in the bladder samples of patients undergoing chemotherapy (Figure $4 \mathrm{~b}$ and $\mathrm{d}$ ).

\section{Differential bladder ICC-LC excitability due to various HCN channel functional statuses}

We performed intracellular $\mathrm{Ca}^{2+}\left(\left[\mathrm{Ca}^{2+}\right]_{\mathrm{i}}\right)$ measurements to evaluate the effects of changes in HCN channel function on 
a

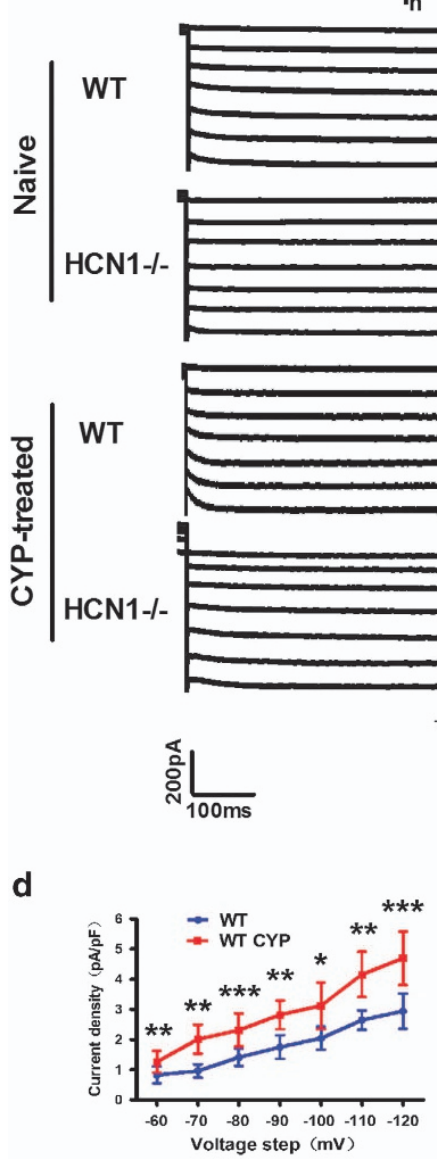

$I_{h}$

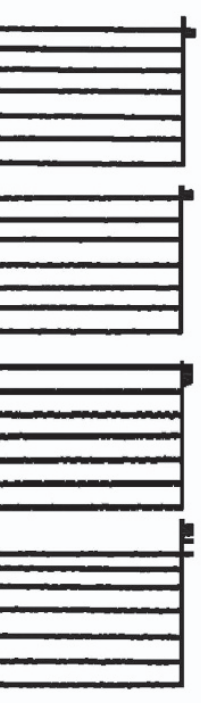

$I_{h}+$ ZD7288

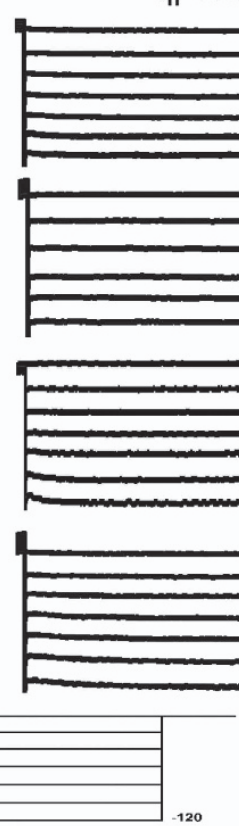

b

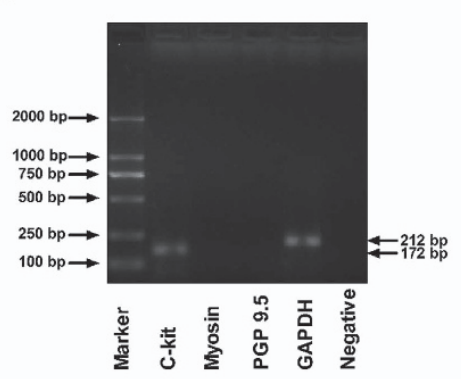

C
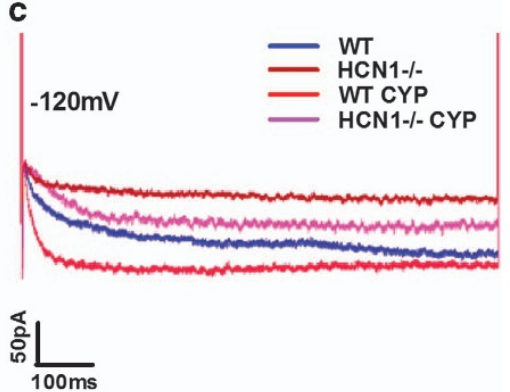

Figure 3 Changes in $/ \mathrm{h}$ properties in bladder ICC-LCs. (a) Characteristic traces of $/ \mathrm{h}$ currents in the bladder ICC-LCs of naive or CYP-treated WT and HCN1 - / - mice evoked by a voltage-clamp protocol with a holding potential of $-60 \mathrm{mV}$ and a step potential ranging from - 60 to $-120 \mathrm{mV}$ in decrements of $10 \mathrm{mV}$. Application of ZD7288 (50 $\mu \mathrm{m})$ reduced $\mathrm{h}_{\mathrm{h}}$ amplitudes in all four groups. (b) RT-PCR showed that only c-kit and GAPDH expression was visualized in the lanes, confirming the reliability of the isolated bladder ICC-LCs. (c) Fully activated current traces of $I_{\mathrm{h}}$ evoked at $-120 \mathrm{mV}$, showing that CYP treatment significantly elevated $I_{\mathrm{h}}$ current amplitudes and HCN channel gating rates in WT and HCN1-/ - mice, while HCN1 channel deletion significantly decreased $\mathrm{I}_{\mathrm{h}}$ current amplitudes and HCN channel gating rates in CYP-treated and naive mice. Over a voltage range of -60 to $-120 \mathrm{mV}$, (d, e) CYP-treatment significantly increased $I_{\mathrm{h}}$ current density in WT and HCN1 - / - mice (data represent the mean \pm s.d., $N=10,{ }^{*} P<0.05$, ${ }^{*} P<0.01$ and ${ }^{* * *} P<0.001$, versus naive mice), $(\mathbf{f}, \mathbf{g})$ while HCN1 channel knockout significantly decreased $I_{\mathrm{h}}$ density in CYP-treated and naive mice (data represent the mean \pm s.d., $N=10,{ }^{*} P<0.05, * * P<0.01$ and ${ }^{* * *} P<0.001$, versus WT mice).

bladder ICC-LC excitability. We compared the responses of bladder ICC-LCs, which were mainly isolated from naive and CYP-treated mice in both genotypes, to stimulation by two pharmaceuticals (CCH and FSK). $\mathrm{CCH}$ administration can simulate the effects of parasympathetic nerve-mediated stimulation, while FSK elevates intracellular cAMP concentrations and activates HCN channels. Stimulation with $\mathrm{CCH}(1 \mu \mathrm{M})$ or FSK $(50 \mu \mathrm{M})$ increased isolated bladder ICC-LC $\left[\mathrm{Ca}^{2+}\right]_{\mathrm{i}}$ in all four groups (Figure $5 \mathrm{a}$ and $\mathrm{b}$ ). Isolated bladder ICC-LCs from CYP-treated mice apparently exhibited enhanced sensitivity to $\mathrm{CCH}$ or FSK compared to isolated bladder ICC-LCs from naive mice in both genotypes. However, the increase in $\left[\mathrm{Ca}^{2+}\right]_{\mathrm{i}}$ provoked by $\mathrm{CCH}$ or FSK in isolated bladder ICC-LCs from CYP-treated WT mice was significantly greater than that provoked by $\mathrm{CCH}$ or FSK in isolated bladder ICC-LCs from CYP-treated HCN1 - / - mice.
HCN1 channel deletion resulted in insensitivity to $\mathrm{CCH}$ or FSK stimulation in isolated bladder ICC-LCs from naive mice (Figure $5 \mathrm{c}-\mathrm{f}$ ). Furthermore, $\left[\mathrm{Ca}^{2+}\right]_{\mathrm{i}}$ in isolated bladder ICC-LCs in all four groups declined upon $50 \mu \mathrm{M}$ ZD7288 application (Figure $5 \mathrm{a}$ and $\mathrm{b}$ ). This finding further validated the authenticity of the isolated bladder ICC-LCs chosen for testing.

\section{Altered detrusor strip contractility ascribed to changes in HCN channel function}

To explore the role of $\mathrm{HCN} 1$ channels in urinary bladder contraction in CYP-induced cystitis, we tested the spontaneous contractions in detrusor strips and the responses of detrusor strips to parasympathetic nerve stimulation simulated by $\mathrm{CCH}$ administration. The phasic amplitudes of the spontaneous contractions in the detrusor strips from CYP-treated mice were significantly increased compared with those from 


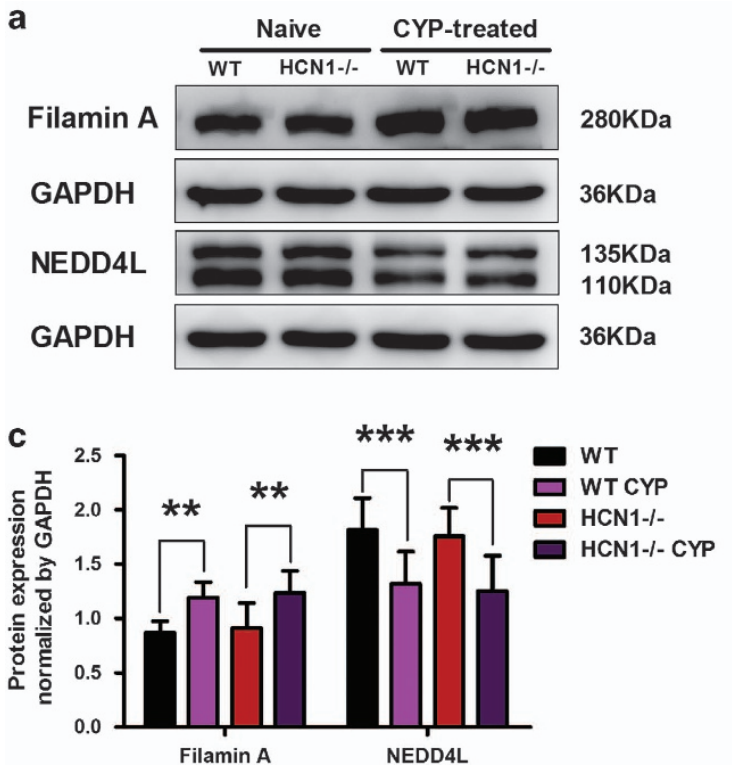

b
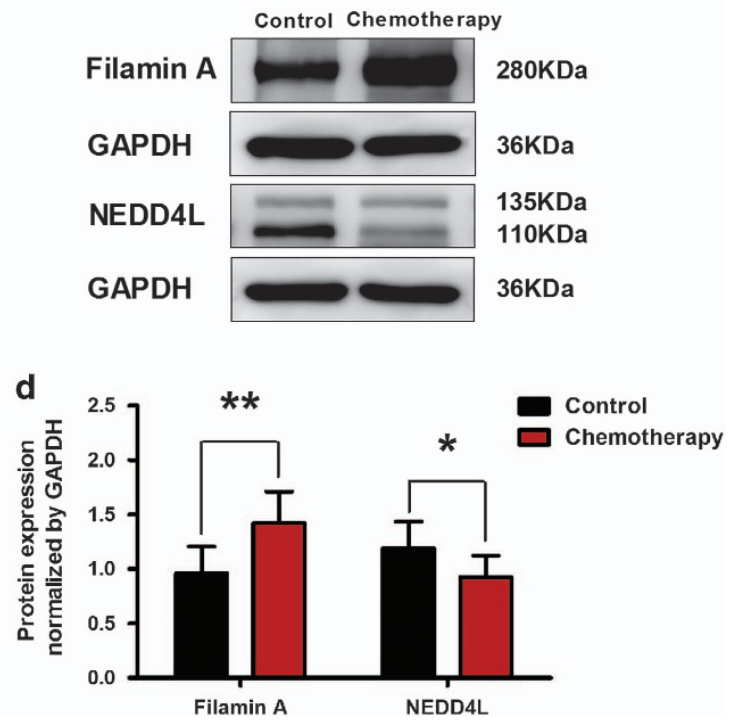

Figure 4 Filamin A and NEDD4L protein expression levels are altered in cystitis. (a, c) Upon CYP treatment, Filamin A protein expression level in the bladder was significantly elevated, while NEDD4L protein expression level was significantly downregulated (data represent the mean \pm s.d., $N=6,{ }^{* *} P<0.01$ and ${ }^{* * *} P<0.001$, versus naive mice). (b, d) The protein expression levels of Filamin A and NEDD4L in the bladder samples of patients undergoing chemotherapy were significantly increased and decreased, respectively, compared with the protein expression levels of Filamin A and NEDD4L in the bladder samples of the control group (data represent the mean $\pm \mathrm{s} . \mathrm{d}$., $N=5,{ }^{*} P<0.05$ and $* * P<0.01$, versus control sample).

naive mice in both genotypes (Figure 6a-c), while the elevations in the phasic amplitudes of the spontaneous contractions induced by CYP treatment were less significant in HCN1 - I - mice than in WT mice (Figure 6a and d). Notably, the phasic amplitudes of the spontaneous contractions in the detrusor strips from CYP-treated $\mathrm{HCN} 1-/-$ mice were almost the same as those from naive WT mice. Furthermore, we found that $\mathrm{HCN} 1$ channel deletion weakened the spontaneous contractions in the detrusor strips from naive mice (Figure $6 a$ and e).

Four cumulative $\mathrm{CCH}$ concentration-response curves were obtained in CYP-treated or naive mice in both genotypes (Figure 6a). Low CCH concentrations $(0.001-0.01 \mu \mathrm{M})$ did not influence the phasic amplitudes of the contractions in detrusor strips in any condition. The amplitudes of the phasic contractile events in detrusor strips induced by high $\mathrm{CCH}$ concentrations $(0.1-10 \mu \mathrm{M})$ were significantly enhanced in CYP-treated mice compared with naive mice in both genotypes, especially the events induced by $1 \mu \mathrm{M} \mathrm{CCH}$ (Figure 6a-c). Likewise, we found that the increases in the amplitudes of the phasic contractions induced by high $\mathrm{CCH}$ concentrations $(0.1-10 \mu \mathrm{M})$ in detrusor strips were less significant in CYP-treated HCN1 - / - mice than in CYP-treated WT mice (Figure 6a and d). Meanwhile, the sensitivity of the detrusor strips of naive mice to high $\mathrm{CCH}$ concentrations $(0.1-10 \mu \mathrm{M})$ was diminished due to the absence of $\mathrm{HCN} 1$ channels (Figure 6a and e).
The development of CYP-induced bladder overactivity was reversed in $\mathrm{HCN} 1-/$ - mice

We performed cystometric recordings to investigate whether HCN1 channels are involved in CYP-induced cystitis-associated bladder dysfunction. We observed the regular voiding cycle and contractility of the bladder in naive WT mice (Figure 7a). Compared to these mice, CYP-treated WT mice exhibited significant urodynamic abnormalities characterized by drastic reductions in the ICI and significant elevations in BP, as well as moderate increases in MBP (Figure 7b-d). Furthermore, the variance in the NVCs was significantly increased in CYP-treated WT mice compared with naive WT mice. Interestingly, we found that the effects of CYP treatment on these urodynamics parameters were less significant in HCN1 - / - mice than in WT mice (Figure $7 \mathrm{~b}-\mathrm{d}$ ). Nevertheless, NVCs were still observed during the filling phase in CYP-treated HCN1 - / - mice (Figure 7a). Moreover, significant differences in urodynamics parameters were noted between naive WT and HCN1 - / - mice. The ICI was moderately extended, and BP and MBP were significantly decreased in HCN1-knockout mice compared with WT mice (Figure $7 \mathrm{~b}-\mathrm{d}$ ).

\section{DISCUSSION}

To our knowledge, this study is the first to reveal that HCN channel expression and function are significantly enhanced in mice with CYP-induced cystitis and that HCN1 channel regulatory proteins are also significantly altered in mice with CYP-induced cystitis. We demonstrated for the first time that HCN1 channel deletion can regulate bladder 
a
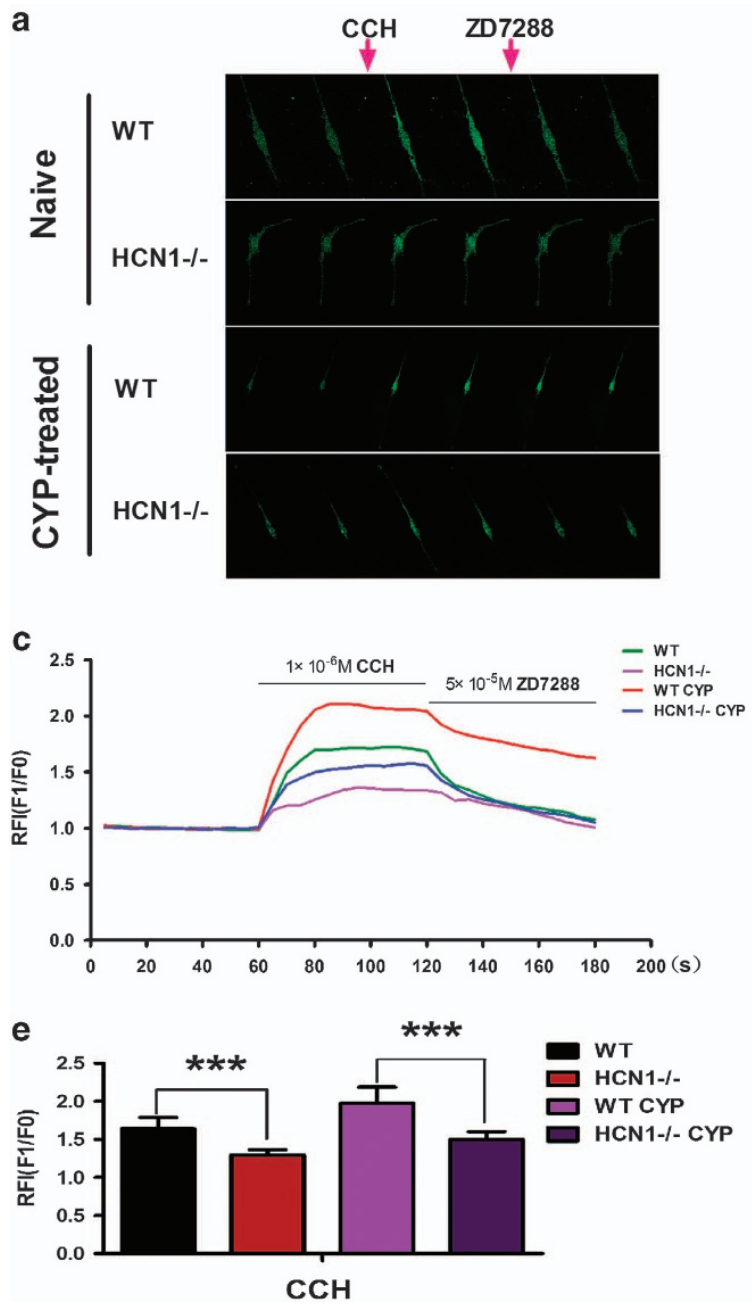

b
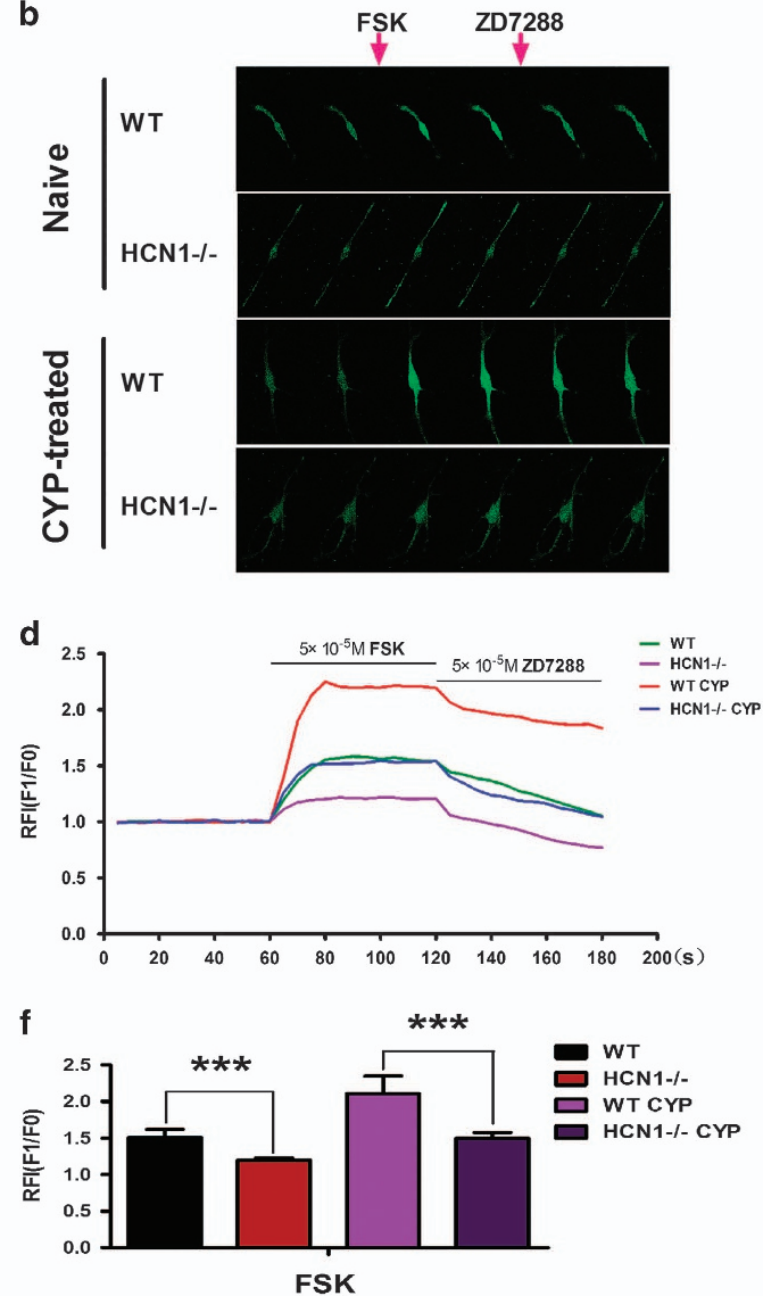

Figure 5 Effects of FSK and $\mathrm{CCH}$ on intracellular calcium ion concentrations $\left(\left[\mathrm{Ca}^{2+}\right]_{\mathrm{i}}\right.$ ) in bladder ICC-LCs. (a, b) Six pictures from each group of naive or CYP-treated WT and $\mathrm{HCN} 1-/-$ mice were chosen to represent the continuous changes in real-time $\left[\mathrm{Ca}^{2+}\right]_{i}$ before and after drug (CCH, FSK, ZD7288) administration. CCH or FSK was applied after recording for 1 min, and ZD7288 was applied after recording for $2 \mathrm{~min}$. (c, d) The effects of $\mathrm{CCH}$ and $\mathrm{FSK}$ on $\left[\mathrm{Ca}^{2+}\right]_{\mathrm{i}}$ in each group were expressed as relative fluorescence intensities (RFI, Fl/FO), which were illustrated as continuous curves. Application of $\mathrm{CCH}(1 \mu \mathrm{M})$ and $\mathrm{FSK}(50 \mu \mathrm{M})$ significantly enhanced $\left[\mathrm{Ca}{ }^{2+}\right]_{\mathrm{i}}$ in all four groups, while application of ZD7288 $(50 \mu \mathrm{m})$ decreased $\left[\mathrm{Ca}^{2+}\right]_{\mathrm{i}}$ in all four groups. HCN1 channel deletion significantly weakened the effects of $\mathrm{CCH}$ (e) or FSK (f) on $\left[\mathrm{Ca}^{2+}\right]_{\mathrm{i}}$ in the bladder ICC-LCs of both naive and CYP-treated mice (data represent the mean \pm s.d., $N=10, * * * P<0.001$, versus WT mice).

function by decreasing bladder ICC-LC excitability and detrusor smooth muscle contractility in both CYP-treated and naive mice. These findings indicate that HCN1 channels in bladder ICC-LCs play a crucial role in regulating bladder function under physiologic conditions and in CYP-induced cystitis.

$\mathrm{HCN}$ channels belong to a superfamily of voltage-gated pore loop channels with four subunits (HCN1-4) in mammals. Increasing amounts of evidence suggest that altered $\mathrm{HCN}$ channels are involved in many excitatory disorders in heterologous systems in addition to the bladder. For example, altered HCN channels are involved in various types of epilepsy, neuropathic pain, Parkinson's disease, ${ }^{18}$ sinoatrial node dysfunction $^{29}$ and Hirschsprung's disease. ${ }^{30}$ These results illustrate that $\mathrm{HCN}$ channels play a requisite role in the pathogenesis of excitatory disorders. The results of the current study indicated that the expression of all four HCN channel subtypes was significantly enhanced in CYP-treated mice compared with naive mice. We obtained identical results with our clinical specimens, indicating that $\mathrm{HCN}$ channels exhibit consistent changes in their expression in inflammatory conditions in various species. We also found that the number of bladder ICC-LCs does not change in cystitis. Therefore, we speculated that the overall increase in HCN channel expression that occurs in cystitis may be due to enhancements of HCN channel expression in single-bladder ICC-LCs. Our corollary was confirmed by patch-clamp experiments, in which we determined that $I_{\mathrm{h}}$ density was significantly increased and that HCN channel gating kinetics were accelerated in individual bladder ICC-LCs from CYP-treated mice. 


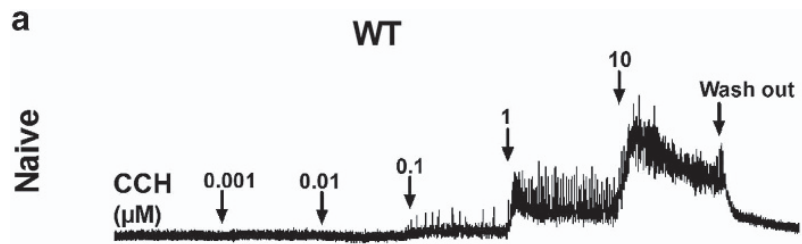

HCN1-/-
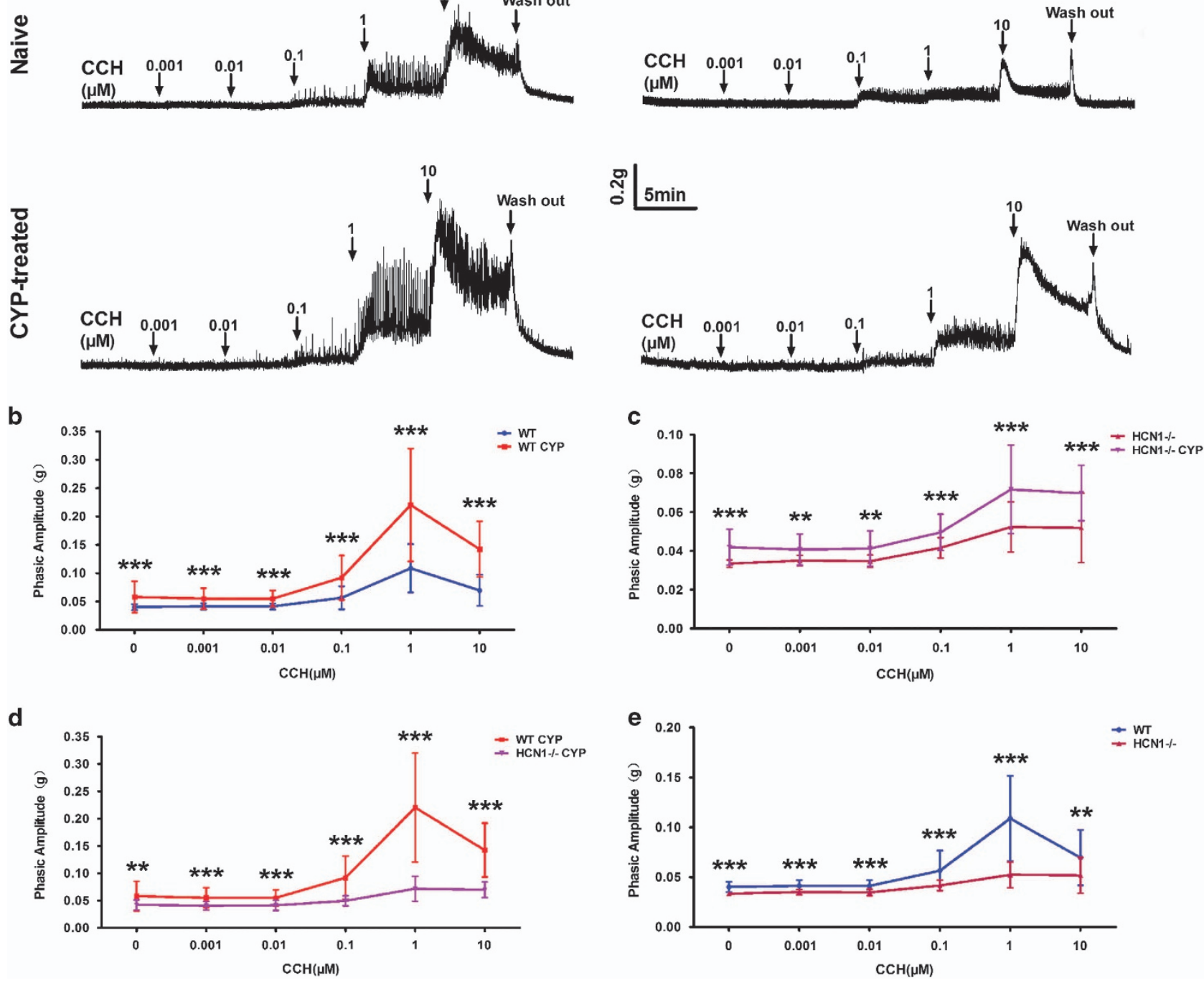

Figure 6 Altered detrusor strip spontaneous contractions and sensitivity to $\mathrm{CCH}$. (a) Detrusor strips isolated from naive or CYP-treated WT and HCN1 - / - mice exhibited different spontaneous contraction amplitudes (before $\mathrm{CCH}$ application) and varied responses to gradient doses of $\mathrm{CCH}(0.001-10 \mu \mathrm{m})$. (b, c) CYP treatment significantly potentiated the spontaneous contractions and $\mathrm{CCH}$-induced phasic contractions of detrusor strips in WT and $\mathrm{HCN1}-/$ - mice (data represent the mean \pm s.d., $N=8$, ${ }^{* *} P<0.01$ and $* * * P<0.001$, versus naive mice). (d, e) HCN1 channel ablation significantly weakened the spontaneous contractions and $\mathrm{CCH}$-induced phasic contractions of detrusor strips in both CYP-treated and naive mice (data represent the mean \pm s.d., $N=8,{ }^{* *} P<0.01$ and ${ }^{* * *} P<0.001$, versus WT mice).

It should be noted that HCN1 channel protein expression was still observed in the bladders of HCN1 - / - mice via western blotting. The HCN1-knockout mice used in our experiments lacked the pore region and S6 transmembrane (pore-S6) domain of the normal HCN1 channel. The HCN1 primary antibody purchased from Abcam targets carboxylterminal amino acids $770-910$ of the HCN1 channel. Therefore, the remaining HCN1 protein fragment (full-length sequence minus the deleted region) would be detected by the HCN1 primary antibody and presented as a band identical to that detected in WT mice. This idea is consistent with the data described by others in a previous study, ${ }^{31}$ in which residual $\mathrm{HCN} 1$ protein fragments in the brains of $\mathrm{HCN} 1-/$ - mice could be detected by both amino-terminal targeting and carboxyl-terminal targeting HCN1 antibodies. HCN1 channel function was definitely greatly affected by $\mathrm{HCN} 1$ protein mutations, which caused significant decreases in $I_{\mathrm{h}}$ density in both CYP-treated and naive mice.

Furthermore, we validated that Filamin A and NEDD4L expression levels were significantly elevated and decreased in cystitis, respectively. Filamin A is known to interact with the $\mathrm{C}$ terminus of the $\mathrm{HCN} 1$ channel via a 22 -amino-acid region and to facilitate HCN1 channel membrane trafficking. ${ }^{32}$ NEDD4L can also interact with the $\mathrm{C}$ terminus of the HCN1 channel to inhibit channel gating and reduce HCN1 channel surface expression..$^{33}$ These findings prompted us to speculate that the above enhancements of $I_{\mathrm{h}}$ density and increases in HCN channel gating rates in CYP-treated WT mice may be 
a

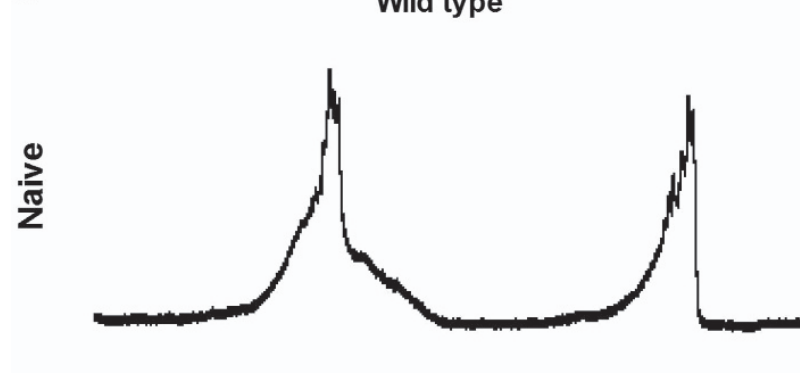

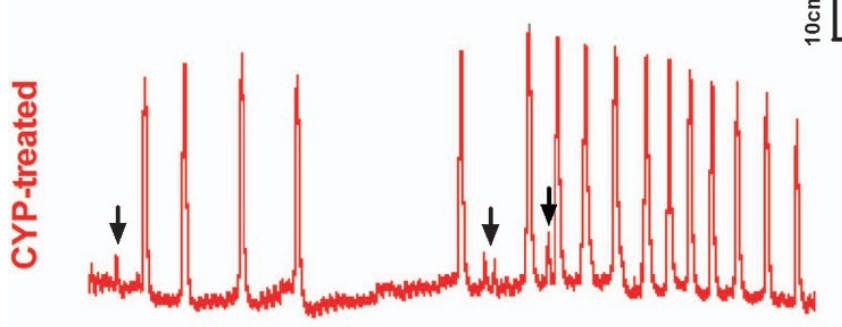

b

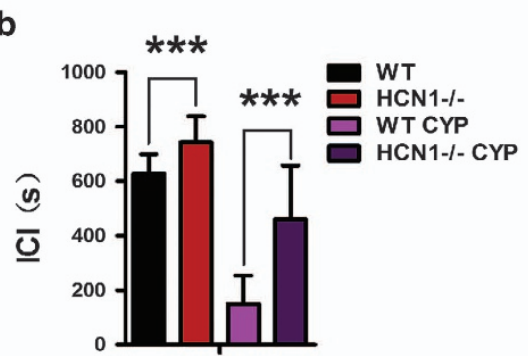

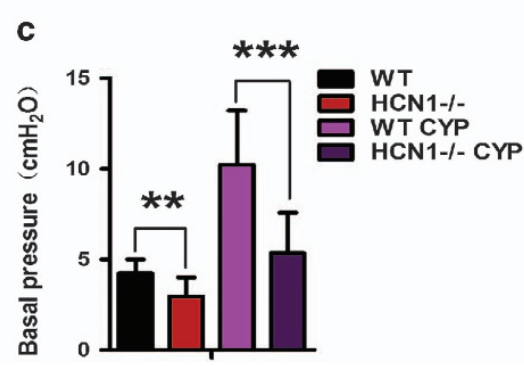

HCN1-/-

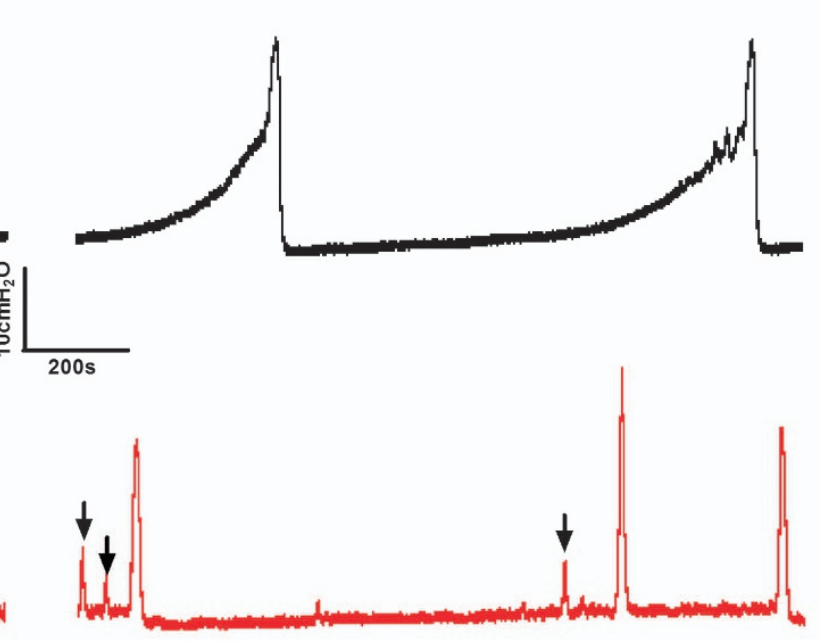

Figure 7 The development of CYP-induced bladder overactivity was reversed in HCN1-/- mice. (a) Various cystometry patterns in naive or CYP-treated WT and HCN1-/- mice. CYP-induced obvious bladder overactivity in WT mice, which was reversed by HCN1 channel deletion. Absence of the HCN1 channel resulted in a relatively hypoactive bladder. Frequent NVCs (black arrow) were observed in both CYP-treated WT and HCN1-/- mice. HCN1 channel knockout significantly increased the ICl (b) and decreased BP (c), MBP (d) in both naive and CYP-treated mice (data represent the mean \pm s.d., $N=8,{ }^{*} P<0.01$ and ${ }^{* * *} P<0.001$, versus WT mice).

partly ascribed to alterations in $\mathrm{HCN} 1$ channel regulatory proteins in the bladder. Based on the above observations, we believe that CYP and other inflammatory factors can disturb $\mathrm{HCN}$ channel regulatory proteins and enhance HCN channel expression and function, which may contribute to the development of cystitis-associated bladder dysfunction.

HCN channels can be opened at a hyperpolarized potential and depolarize the membrane by generating an inward current consisting of $\mathrm{Na}^{+}$and $\mathrm{K}^{+18}$. The effects of HCN channels on cell membrane electrophysiology have been validated in several types of cells. It has been reported that HCN channels mediate spontaneous pacemaker potentials in cultured mouse colon $\mathrm{ICCs}^{20}$ and that $\mathrm{HCN}$ channels can trigger action potentials in sinoatrial node myocytes and thalamocortical relay neurons by generating pacemaker depolarization, resulting in the activation of low-voltage-activated $\mathrm{Ca}^{2+}$ channels, such as T-type or L-type $\mathrm{Ca}^{2+}$ channels, ${ }^{34}$ leading to a remarkable $\mathrm{Ca}^{2+}$ influx and facilitating cell excitation.

Therefore, to explore whether $\mathrm{Ca}^{2+}$ influx in bladder ICC-LCs is influenced by alterations in HCN channel expression and function, we conducted $\left[\mathrm{Ca}^{2+}\right]_{\mathrm{i}}$ measurements to test the effects of FSK and CCH on bladder ICC-LCs, which enabled us to evaluate the excitability of the bladder ICC-LCs in each group. To our knowledge, cAMP can activate HCN channels by directly binding to their cyclic nucleotide-binding domain. ${ }^{18}$ FSK elevates intracellular cAMP concentrations and activates $\mathrm{HCN}$ channels. ${ }^{35} \mathrm{CCH}$, an analog of acetylcholine, can activate muscarinic cholinergic receptors and simulate the effects of parasympathetic nerve stimulation. ${ }^{36}$ Our previous study demonstrated that bladder ICC-LCs express $M_{2}$ and $M_{3}$ cholinergic receptors; however, the latter receptors are more important for mediating bladder ICC-LC excitability than the former receptors. ${ }^{37} \mathrm{~A}$ previous study showed that $M_{1}$ cholinergic receptor excitation leads to a positive shift in the $\mathrm{HCN}$ channel activation curve through a $\mathrm{PIP}_{2}$ synthesis upregulation-dependent mechanism. ${ }^{38}$ Given that both $M_{1}$ and $M_{3}$ cholinergic receptors are coupled to $\mathrm{G}_{\mathrm{q} / 11},{ }^{39}$ we presume that $\mathrm{M}_{3}$ cholinergic receptor activation can facilitate bladder ICC-LC HCN channel gating by the same mechanism by which $\mathrm{M}_{1}$ cholinergic receptors facilitate HCN channel gating, but additional studies are required to elucidate the exact mechanism underlying this phenomenon. In our study, we found that FSK and CCH stimulated obvious $\mathrm{Ca}^{2+}$ influx in bladder ICC-LCs, but the amplitudes of 
$\mathrm{Ca}^{2+}$ influx varied among all four groups. Notably, HCN channel ablation significantly attenuated bladder ICC-LC activity in CYP-treated and naive mice. Therefore, we believe that the differential sensitivity of bladder ICC-LCs to FSK or $\mathrm{CCH}$ can be ascribed to variations in the functional statuses of the HCN channels in each group. In addition, based on the results of our patch-clamp experiment, in which we observed that the HCN1 channel accounts for approximately one-third of the total $\mathrm{HCN}$ message in both CYP-treated and naive mice, we surmised that the HCN1 channel plays a more dominant role than other HCN channels in regulating bladder ICC-LC activity in both physiological and inflammatory conditions.

Isolated detrusor strips possess the capacity to spontaneously contract in vitro, a behavior that has been confirmed to be controlled by bladder ICC-LCs. ${ }^{40}$ In the current study, in response to diverse bladder ICC-LC excitability levels, we determined that the detrusor strips of CYP-treated mice exhibited the strongest spontaneous contractions, and these contractions were significantly weakened by $\mathrm{HCN} 1$ channel deletion in CYP-treated and naive mice. Furthermore, using urodynamic measurements, we found that CYP-induced bladder dysfunction was significantly reversed by $\mathrm{HCN} 1$ channel deletion, and naive HCN1-knockout mice exhibited relatively hypoactive bladders. These results indicate that HCN channels, especially HCN1 channels, can modulate the spontaneous contractions of detrusor strips by controlling bladder ICC-LC excitability to regulate bladder function in both physiological and inflammatory conditions. Moreover, it also has been shown that bladder ICC-LCs have close structural relationships with cholinergic nerves ${ }^{41}$ and that bladder ICC-LCs are considered powerful transducers of the signals between nerve fibers and detrusor myocytes. ${ }^{42}$ In our study, we found that HCN1 channel ablation decreased the responses of detrusor strips to gradient doses of $\mathrm{CCH}$. Given these results and the results of our $\left[\mathrm{Ca}^{2+}\right]_{i}$ measurement experiments that bladder ICC-LCs lacking the HCN1 channels exhibited reduced responses to $\mathrm{CCH}$, we also speculate that the $\mathrm{HCN} 1$ channels may regulate the processes of receiving, integrating and transmitting neural signals in bladder ICC-LCs and harmonize detrusor smooth muscle contractions. Alterations in these processes caused by changes in HCN1 channel expression and function may also be involved in the pathogenesis of CYP-induced cystitis.

Thus far, the precise pathogenesis of IC/BPS has not been well defined. ${ }^{43}$ Our findings will serve as a potent supplement to the existing literature with respect to explaining the exact pathogenesis of IC/BPS. In addition, our findings suggest that the HCN1 channels in bladder ICC-LCs can be regarded as a valid therapeutic target for ameliorating LUTS in patients with IC/BPS or cystitis induced by chemotherapy or radiotherapy. A limitation of our study is that we only harvested the bladder ICC-LCs in the detrusor for study, so that the roles of bladder ICC-LCs in the lamina propria could not be investigated. The approach used in this study should be improved to harvest the bladder ICC-LCs in the lamina propria in further studies. Furthermore, in the future, knockout mice lacking other HCN subtypes may be used in studies to explore the possible roles of these other HCN subtypes in CYP-induced cystitis or other types of bladder dysfunction. Moreover, a series of additional studies are required to clarify the exact mechanisms by which HCN channels are regulated in cystitis.

In conclusion, the present study has demonstrated that CYP can induce $\mathrm{HCN}$ channel regulatory protein dysfunction and then upregulate $\mathrm{HCN}$ channel expression and function in bladder ICC-LCs. Furthermore, we found that HCN1 channel deletion can regulate bladder function by decreasing bladder ICC-LC activity and detrusor smooth muscle contractility in both physiological and inflammatory conditions. Therefore, we believe that CYP-induced enhancements of HCN1 channel expression in bladder ICC-LCs are essential for cystitisassociated bladder hyperactivity and that the HCN1 channel is a viable therapeutic target for managing bladder hyperactivity.

\section{CONFLICT OF INTEREST}

The authors declare no conflict of interest.

\section{ACKNOWLEDGEMENTS}

This work was supported by the National Natural Science Foundation of China (Nos 81300630 and 81230017).

Author contributions: QL performed the experiments and drafted the manuscript. ZL and XYD analyzed the data. TZ, BSS, JL and JZ participated in the H\&E staining and the western blotting and immunofluorescence analyses. JZZ, QQW, ZXY and XYH were involved in the patch-clamp experiments, $\left[\mathrm{Ca}^{2+}\right]_{\mathrm{i}}$ measurements, contractility studies and urodynamic measurements. LKL designed the research and edited the paper. All authors reviewed and approved the submission of the paper.

1 Everaerts W, Zhen X, Ghosh D, Vriens J, Gevaert T, Gilbert JP et al. Inhibition of the cation channel TRPV4 improves bladder function in mice and rats with cyclophosphamide-induced cystitis. Proc Natl Acad Sci USA 2010; 107: 19084-19089.

2 Andersson KE, Arner A. Urinary bladder contraction and relaxation: physiology and pathophysiology. Physiol Rev 2004; 84: 935-986.

3 Kairys AE, Schmidt-Wilcke T, Puiu T, Ichesco E, Labus JS, Martucci K et al. Increased brain gray matter in the primary somatosensory cortex is associated with increased pain and mood disturbance in patients with interstitial cystitis/painful bladder syndrome. J Urol 2015; 193: 131-137.

4 Humphrey L, Arbuckle R, Moldwin R, Nordling J, van de Merwe JP, Meunier J et al. The bladder pain/interstitial cystitis symptom score: development, validation, and identification of a cut score. Eur Urol 2012; 61: 271-279.

5 Kuo YC, Kuo HC. Potential factors that can be used to differentiate between interstitial cystitis/painful bladder syndrome and bladder oversensitivity in women. Int J Clin Pract 2012; 66: 146-151.

6 Coelho A, Wolf-Johnston AS, Shinde S, Cruz CD, Cruz F, Avelino A et al. Urinary bladder inflammation induces changes in urothelial nerve growth factor and TRPV1 channels. Br J Pharmacol 2015; 172: 1691-1699.

7 Monjotin N, Gillespie J, Farrié M, Le Grand B, Junquero D, Vergnolle N. F16357, a novel protease-activated receptor 1 antagonist, improves urodynamic parameters in a rat model of interstitial cystitis. Br J Pharmacol 2016; 173: 2224-2236.

8 Boudes M, Uvin P, Kerselaers S, Vennekens R, Voets T, De Ridder D. Functional characterization of a chronic cyclophosphamide-induced overactive bladder model in mice. Neurourol Urodyn 2011; 30: 1659-1665.

9 Freitas RD, Costa KM, Nicoletti NF, Kist LW, Bogo MR, Campos MM. Omega-3 fatty acids are able to modulate the painful symptoms associated 
to cyclophosphamide-induced-hemorrhagic cystitis in mice. J Nutr Biochem 2016; 27: 219-232.

10 Gfroerer S, Rolle U. Interstitial cells of Cajal in the normal human gut and in Hirschsprung disease. Pediatr Surg Int 2013; 29: 889-897.

11 Sanders KM, Ward SM, Koh SD. Interstitial cells: regulators of smooth muscle function. Physiol Rev 2014; 94: 859-907.

12 He P, Deng J, Zhong X, Zhou Z, Song B, Li L. Identification of a hyperpolarization-activated cyclic nucleotide-gated channel and its subtypes in the urinary bladder of the rat. Urology 2012; 79: 1411.e7-1411.13.

13 Deng J, He P, Zhong X, Wang Q, Li L, Song B. Identification of T-type calcium channels in the interstitial cells of Cajal in rat bladder. Urology 2012; 80: 1389.e1-1389.e7.

14 Anderson UA, Carson C, McCloskey KD. KCNQ currents and their contribution to resting membrane potential and the excitability of interstitial cells of Cajal from the guinea pig bladder. J Urol 2009; 182: 330-336.

15 McCloskey KD, Gurney AM. Kit positive cells in the guinea pig bladder. J Urol 2002; 168: 832-836.

16 McCloskey KD. Bladder interstitial cells: an updated review of current knowledge. Acta Physiol 2013; 207: 7-15.

17 Gevaert T, De Vos R, Everaerts W, Libbrecht L, Van Der Aa F, van den Oord $\mathrm{J}$ et al. Characterization of upper lamina propria interstitial cells in bladders from patients with neurogenic detrusor overactivity and bladder pain syndrome. J Cell Mol Med 2011; 15: 2586-2593.

18 He C, Chen F, Li B, Hu Z. Neurophysiology of HCN channels: from cellular functions to multiple regulations. Prog Neurobiol 2014; 112: 1-23.

19 Wahl-Schott C, Biel M. HCN channels: structure, cellular regulation and physiological function. Cell Mol Life Sci 2009; 66: 470-494.

20 Shahi PK, Choi S, Zuo DC, Kim MY, Park CG, Kim YD et al. The possible roles of hyperpolarization-activated cyclic nucleotide channels in regulating pacemaker activity in colonic interstitial cells of Cajal. J Gastroenterol 2014; 49: 1001-1010.

21 Dong X, Song Q, Zhu J, Zhao J, Liu Q, Zhang T et al. Interaction of Caveolin-3 and $\mathrm{HCN}$ is involved in the pathogenesis of diabetic cystopathy. Sci Rep 2016; 6: 24844.

22 Deng T, Zhang Q, Wang Q, Zhong X, Li L. Changes in hyperpolarizationactivated cyclic nucleotide-gated channel expression and activity in bladder interstitial cells of Cajal from rats with detrusor overactivity. Int Urogynecol J 2015; 26: 1139-1145.

23 Fischer AH, Jacobson KA, Rose J, Zeller R. Hematoxylin and eosin staining of tissue and cell sections. CSH Protoc 2008; 2008: pdb.prot4986.

24 Wang Y, Fang Q, Lu Y, Song B, Li W, Li L. Effects of mechanical stretch on interstitial cells of Cajal in guinea pig bladder. J Surg Res 2010; 164: e213-e219.

25 Davidson RA, McCloskey KD. Morphology and localization of interstitial cells in the guinea pig bladder: structural relationships with smooth muscle and neurons. J Urol 2005; 173: 1385-1390.

26 Zhong X, Deng J, He P, You N, Wang Q, Song B et al. Reverse mode of the sodium/calcium exchanger subtype 3 in interstitial cells of Cajal from rat bladder. Urology 2013; 82: 254.e7-254.e12.

27 Andersson KE, Soler R, Füllhase C. Rodent models for urodynamic investigation. Neurourol Urodyn 2011; 30: 636-646.

28 Gevaert T, Vriens J, Segal A, Everaerts W, Roskams T, Talavera K et al. Deletion of the transient receptor potential cation channel TRPV4 impairs murine bladder voiding. J Clin Invest 2007; 117: 3453-3462.

29 Yeh YH, Burstein B, Qi XY, Sakabe M, Chartier D, Comtois P et al. Funny current downregulation and sinus node dysfunction associated with atrial tachyarrhythmia: a molecular basis for tachycardia-bradycardia syndrome. Circulation 2009; 119: 1576-1585.
30 O'Donnell AM, Coyle D, Puri P. Decreased expression of hyperpolarisationactivated cyclic nucleotide-gated channel 3 in Hirschsprung's disease. World J Gastroenterol 2015; 21: 5635-5640.

31 Ramakrishnan NA, Drescher MJ, Khan KM, Hatfield JS, Drescher DG. HCN1 and $\mathrm{HCN} 2$ proteins are expressed in cochlear hair cells: HCN1 can form a ternary complex with protocadherin $15 \mathrm{CD} 3$ and F-actin-binding filamin A or can interact with HCN2. J Biol Chem 2012; 287: 37628-37646.

32 Gravante B, Barbuti A, Milanesi R, Zappi I, Viscomi C, DiFrancesco D. Interaction of the pacemaker channel HCN1 with filamin A. J Biol Chem 2004; 279: 43847-43853.

33 Wilkars W, Wollberg J, Mohr E, Han M, Chetkovich DM, Bähring R et al. Nedd4-2 regulates surface expression and may affect $\mathrm{N}$-glycosylation of hyperpolarization-activated cyclic nucleotide-gated (HCN)-1 channels. FASEB J 2014; 28: 2177-2190.

34 Robinson RB, Siegelbaum SA. Hyperpolarization-activated cation currents: from molecules to physiological function. Annu Rev Physiol 2003; 65 : 453-480.

35 Du L, Wang SJ, Cui J, He WJ, Ruan HZ. The role of HCN channels within the periaqueductal gray in neuropathic pain. Brain Res 2013; 1500: 36-44.

36 Thorneloe KS, Meredith AL, Knorn AM, Aldrich RW, Nelson MT. Urodynamic properties and neurotransmitter dependence of urinary bladder contractility in the BK channel deletion model of overactive bladder. Am J Physiol Renal Physiol 2005; 289: F604-F610.

37 Wu Y, Shi C, Deng J, Zhang X, Song B, Li L. Expression and function of muscarinic subtype receptors in bladder interstitial cells of cajal in rats. Urol J 2014; 11: 1642-1647.

38 Pian P, Bucchi A, Decostanzo A, Robinson RB, Siegelbaum SA. Modulation of cyclic nucleotide-regulated $\mathrm{HCN}$ channels by $\mathrm{PIP}(2)$ and receptors coupled to phospholipase C. Pflugers Arch 2007; 455: 125-145.

39 Akam EC, Challiss RA, Nahorski SR. $\mathrm{G}_{\mathrm{q} / 11)}$ and G(i/o) activation profiles in $\mathrm{CHO}$ cells expressing human muscarinic acetylcholine receptors: dependence on agonist as well as receptor-subtype. Br J Pharmacol 2001; 132: 950-958.

40 Vahabi B, McKay NG, Lawson K, Sellers DJ. The role of c-kit-positive interstitial cells in mediating phasic contractions of bladder strips from streptozotocin-induced diabetic rats. BJU Int 2011; 107: 1480-1487.

41 McCloskey KD. Interstitial cells in the urinary bladder-localization and function. Neurourol Urodyn 2010; 29: 82-87.

42 Juszczak K, Maciukiewicz P, Drewa T, Thor PJ. Cajal-like interstitial cells as a novel target in detrusor overactivity treatment: true or myth? Cent Eur J Urol 2014; 66: 413-417.

43 Nasrin S, Masuda E, Kugaya H, Ito Y, Yamada S. Improvement by phytotherapeutic agent of detrusor overactivity, down-regulation of pharmacological receptors and urinary cytokines in rats with cyclophosphamide induced cystitis. J Urol 2013; 189: 1123-1129.

This work is licensed under a Creative Commons Attribution-NonCommercial-NoDerivs 4.0 International License. The images or other third party material in this article are included in the article's Creative Commons license, unless indicated otherwise in the credit line; if the material is not included under the Creative Commons license, users will need to obtain permission from the license holder to reproduce the material. To view a copy of this license, visit http://creativecommons.org/licenses/by-nc-nd/4.0/ 